Technical Note

\title{
Slow delivery of a nitrification inhibitor (dicyandiamide) to soil using a biodegradable hydrogel of chitosan
}

\author{
E.P. Minet ${ }^{\mathrm{a}, *}$, C. O'Carroll ${ }^{\mathrm{b}}$, D. Rooney ${ }^{\mathrm{b}}$, C. Breslin ${ }^{\mathrm{b}}$, C.P. McCarthy ${ }^{\mathrm{b}}$, L. Gallagher ${ }^{\mathrm{b}}$, K.G. Richards ${ }^{\mathrm{a}, *}$ \\ ${ }^{a}$ Teagasc Environment Research Centre, Johnstown Castle, Co. Wexford, Ireland \\ ${ }^{\mathrm{b}}$ National University of Ireland Maynooth, Chemistry Department, Maynooth, Co. Kildare, Ireland
}

\section{H I G H L I G H T S}

- Dicyandiamide (DCD) was encapsulated in glyoxal-crosslinked chitosan hydrogel beads.

- Chitosan delayed the release of nitrification inhibitor DCD in water and soil.

- DCD release was controlled by glyoxal polymerisation inside chitosan.

- The higher glyoxal polymerisation the more delayed DCD release in water or in soil.

- The higher glyoxal polymerisation the less DCD encapsulated in the beads.

\section{A R T I C L E I N F O}

\section{Article history:}

Received 8 May 2013

Received in revised form 29 July 2013

Accepted 13 August 2013

Available online 12 September 2013

\section{Keywords:}

Chitosan

Hydrogel

Dicyandiamide

Slow release

Nitrification inhibitor

Nitrogen loss

\begin{abstract}
A B S T R A C T
Using chemical inhibitors to reduce soil nitrification decreases emissions of environmental damaging nitrate and nitrous oxide and improves nitrogen use efficiency in agricultural systems. The efficacy of nitrification inhibitors such as dicyandiamide (DCD) is limited in soil due to biodegradation. This study investigated if the persistence of DCD could be sustained in soil by slow release from a chitosan hydrogel. DCD was encapsulated in glyoxal-crosslinked chitosan beads where excess glyoxal was (i) partly removed ( $C$ beads) or (ii) allowed to dry (CG beads). The beads were tested in water and in soil. The beads contained two fractions of DCD: one which was quickly released in water, and one which was not. A large DCD fraction within C beads was readily available: $84 \%$ of total DCD bead content was released after $9 \mathrm{~h}$ immersion in water, while between $74 \%$ and $98 \%$ was released after $7 \mathrm{~d}$ in soil under low to high moisture conditions. A lower percentage of encapsulated DCD was readily released from CG beads: $19 \%$ after $9 \mathrm{~h}$ in water, and 33\% after $7 \mathrm{~d}$ in soil under high rainfall conditions. Kinetic analysis indicated that the release in water occurred by quasi-Fickian diffusion. The results also suggest that DCD release was controlled by bead erosion and the leaching of glyoxal derivatives, predominantly a glyoxal-DCD adduct whose release was positively correlated with that of $\mathrm{DCD}\left(R^{2}=0.99, p \leqslant 0.0001\right)$. Therefore, novel chitosan/glyoxal composite beads show a promising slow-release potential in soil for agrochemicals like DCD.
\end{abstract}

(c) 2013 Elsevier Ltd. All rights reserved.

\section{Introduction}

Reactive $\mathrm{N}$ losses and greenhouse gas (GHG) emissions from agricultural soils are an important source of water and air pollution (Stark and Richards, 2008a). In this contamination process, $\mathrm{NH}_{4}^{+}$ plays a central role. To date, national and international green legislation has aimed to control such $\mathrm{N}$ losses and GHG emissions but with limited success (Stark and Richards, 2008b). To compensate

\footnotetext{
* Corresponding authors. Tel.: +353 539171261; fax: +353 539142213 (K.G. Richards).

E-mail addresses: eddy.minet@gmail.com (E.P. Minet),karl.richards@teagasc.ie (K.G. Richards).
}

for these limitations, researchers have investigated complementary $\mathrm{N}$ mitigation techniques, such as blocking $\mathrm{NH}_{4}^{+}$oxidation to $\mathrm{NO}_{3}^{-}$using nitrification inhibitors (Yu et al., 2007; Watson et al., 2009). Studies have revealed that the inhibitory potential of dicyandiamide (DCD) can curb nitrate leaching by up to 76\% (Di and Cameron, 2004) and $\mathrm{N}_{2} \mathrm{O}$ emissions by up to $70 \%$ (Di et al., 2007). Yet for all its effectiveness, degradation in soil limits the persistence of DCD (Estermaier et al., 1992), which has a half-life estimated between $110 \mathrm{~d}$ at $5{ }^{\circ} \mathrm{C}$ and approximately $20 \mathrm{~d}$ at $25^{\circ} \mathrm{C}$ (Kelliher et al., 2008). Consequently, repeated soil applications are required to maintain efficacy.

The ephemeral subsistence of agrochemicals applied to soils is a recurrent problem that affects a large range of products. 
Attempts to overcome this drawback have seen the emergence of controlled release formulations (CRF) (Fernández-Pérez et al., 2001), whereby active compounds are encapsulated in a slow release matrix prior to soil application. The expected advantages of this approach include: (i) prolonged activity in soil (protection from microbial degradation until release), (ii) reduced number of applications, (iii) reduced costs (due to single application) and (iv) reduced environmental loss (Akelah, 1996). A CRF with some interesting potential is chitosan, a polymeric hydrogel well known for its slow drug delivery use in the pharmaceutical industry (Rinaudo, 2006). This non-toxic and biodegradable copolymer of $\beta$-(1 $\rightarrow 4)$-linked 2-acetamido-2-deoxy-D-glucopyranose and 2-amino-2-deoxy-D-glucopyranose results from the partial deacetylation of chitin, which is the main component of the exoskeleton of crustaceans. Like all hydrogels, chitosan is highly porous and hydrophilic which allows a high rate of wetting. This permits an encapsulated compound to diffuse out at a speed which is controlled by the crosslinking density and pore size (Berger et al., 2004). To the best of our knowledge slow release of DCD has only been attempted by Bishop (2010). Moreover, very few studies have considered the use of chitosan-derived systems for the controlled delivery of agrochemicals. Some notable examples include the delivery of neem seed oil (Devi and Maji, 2009), plant hormones (Quiñones et al., 2010; Fan et al., 2012) and fertilisers and herbicide (Teixeira et al., 1990).

The objective of our study was to explore for the first time the effect of a slow release system (chitosan xerogel beads loaded with dicyandiamide) on DCD soil release. An underlying purpose was to assess the potential of chitosan encapsulation as a credible alternative to repeated soil applications of DCD.

\section{Experimental}

DCD was encapsulated in chitosan hydrogel beads formed by precipitation of an acidified chitosan gelling solution and covalently crosslinked with glyoxal. In these beads, excess glyoxal was (i) partly removed by washing with an aqueous solution of DCD (60 mM) (C beads) or (ii) allowed to dry (CG beads) (see Method SM-1 for details). To estimate the amount of DCD encapsulated in C and CG beads, DCD was extracted with acidified and unacidified deionised water (Method SM-2). DCD release from the beads, kinetics of that release and swelling ratio $\left(S_{w} \%\right)$ were investigated in water over $2 \mathrm{wk}$ incubations (15 min up to $14 \mathrm{~d}$ ) (Method SM-3). DCD soil release from $C$ beads was assessed under two treatments (rainfall, soil moisture expressed as water holding capacity (WHC)), three rates of added water and three incubation times (up to $7 \mathrm{~d}$ ) (Method SM-4). DCD soil release from CG beads was assessed under the highest rate of rainfall only.

Bead morphology and surface analyses were carried out with a scanning electron microscope (SEM). The DCD content in water samples and $\mathrm{KCl}$ soil extracts was quantified by HPLC analysis according to a modified method by Turowski and Deshmukh (2004) (Method SM-5).

The effects of independent variables (bead type and DCD extraction method in Section 3.1.2; soil treatment, treatment rates and incubation time for DCD soil release from $C$ beads in Section 3.2) and their interactions on response variables (amount of DCD encapsulated in beads or released in soil in Sections 3.1.2 and 3.2 respectively) were investigated by ANOVA after model assumptions were met. When significant effects were found $(p \leqslant 0.05)$, Bonferroni Post Hoc tests were used to make pairwise comparisons. Parametric tests (correlations, linear and non-linear regressions) were carried out to explore some relationships between continuous variables.

\section{Results and discussions}

\subsection{Beads characterisation}

\subsubsection{Bead morphology and surface analysis}

SEM micrographs of lightly washed $C$ and CG beads are presented in Fig. 1. The beads are distorted spheres, with dimensions between 1.5 and $2 \mathrm{~mm}$. Unlike CG beads, the surface morphology of the $C$ beads was quite wrinkled. Both bead types had a highly compact interior showing no pores with diameters in the micrometer range. The interior of the CG beads appeared more fibrous in nature compared to that of the $C$ beads. Despite all beads being made from the same amount of chitosan, the initial dry weight of CG beads ( $4.408 \pm 0.027 \mathrm{mg}$ per bead) was $46 \%$ higher than that of $C$ beads ( $3.090 \pm 0.009 \mathrm{mg}$ per bead). The increased weight of the CG beads was attributed to the excess glyoxal, which can undergo a number of reactions within the beads. Glyoxal is known to undergo oligomerisation upon loss of water and it can easily polymerise in the solid state (Loeffler et al., 2006; Nakajima et al., 2007). In addition, it is possible that glyoxal could react with the guanidine group of the DCD to form Schiff base glyoxal-DCD adducts (Panicucci and McClelland, 1989). These excess glyoxal compounds were partly removed from the $\mathrm{C}$ beads before the drying step by washing in a solution containing DCD.

\subsubsection{DCD content within the beads}

Immersing the beads in deionised or acidified water was used to extract the DCD from the hydrogel and the quantity extracted was measured by HPLC analysis. This study revealed that $C$ beads contained significantly more $(p \leqslant 0.0001)$ DCD (about $0.2 \mathrm{mg}$ DCD per bead depending on the extraction method) than CG beads (less than $0.05 \mathrm{mg}$ DCD per bead) (Fig. 2a). Acidification significantly increased $(p \leqslant 0.0001)$ the amount of DCD extracted when compared with immersion in deionised water. It is likely that the acidification causes an increase in DCD release from the CG beads due to two factors. Acidification caused the beads to swell considerably and therefore more DCD could diffuse out of the beads. Moreover, it is possible that glyoxal-DCD adducts could be hydrolysed under acidic conditions to re-form DCD. A study involving mechanical destruction of the beads coupled with acidification confirmed that the total content of DCD in the beads was reliably obtained by simply incubating uncrushed beads in acidified water for $2 \mathrm{wk}(0.219$ and $0.043 \mathrm{mg}$ DCD per C and CG beads respectively). The incubation of DCD solutions under similar conditions confirmed the stability of the molecule, so DCD extracted from beads was assumed to truly reflect the amount encapsulated. The fact that not all the DCD was released from the beads after prolonged immersion in deionised water implied the existence of two DCD fractions in the beads: available DCD which can easily diffuse out during water incubation, and locked DCD which remains trapped in the beads.

Besides DCD, a second compound with a HPLC retention time of $4.6 \mathrm{~min}$ was detected in beads incubated in water (no other meaningful peak was detected between 190 and $300 \mathrm{~nm}$ during the 10 min run). The peak did not correspond to those observed for the starting glyoxal solution. It was detected only when both DCD and glyoxal were present within the beads (no peak was detected when beads were prepared without glyoxal or without DCD). The destruction of beads by acidification released about twice as much of this glyoxal derivative compared to incubation in deionised water (Fig. 2b). This clearly indicates that this compound, probably a glyoxal-DCD adduct, does not undergo facile acid hydrolysis to release more DCD. As can be seen from Fig. 2b, CG beads released 54\% more glyoxal-DCD adduct than $C$ beads when acidified (53\% with acidified crushed beads), which is 

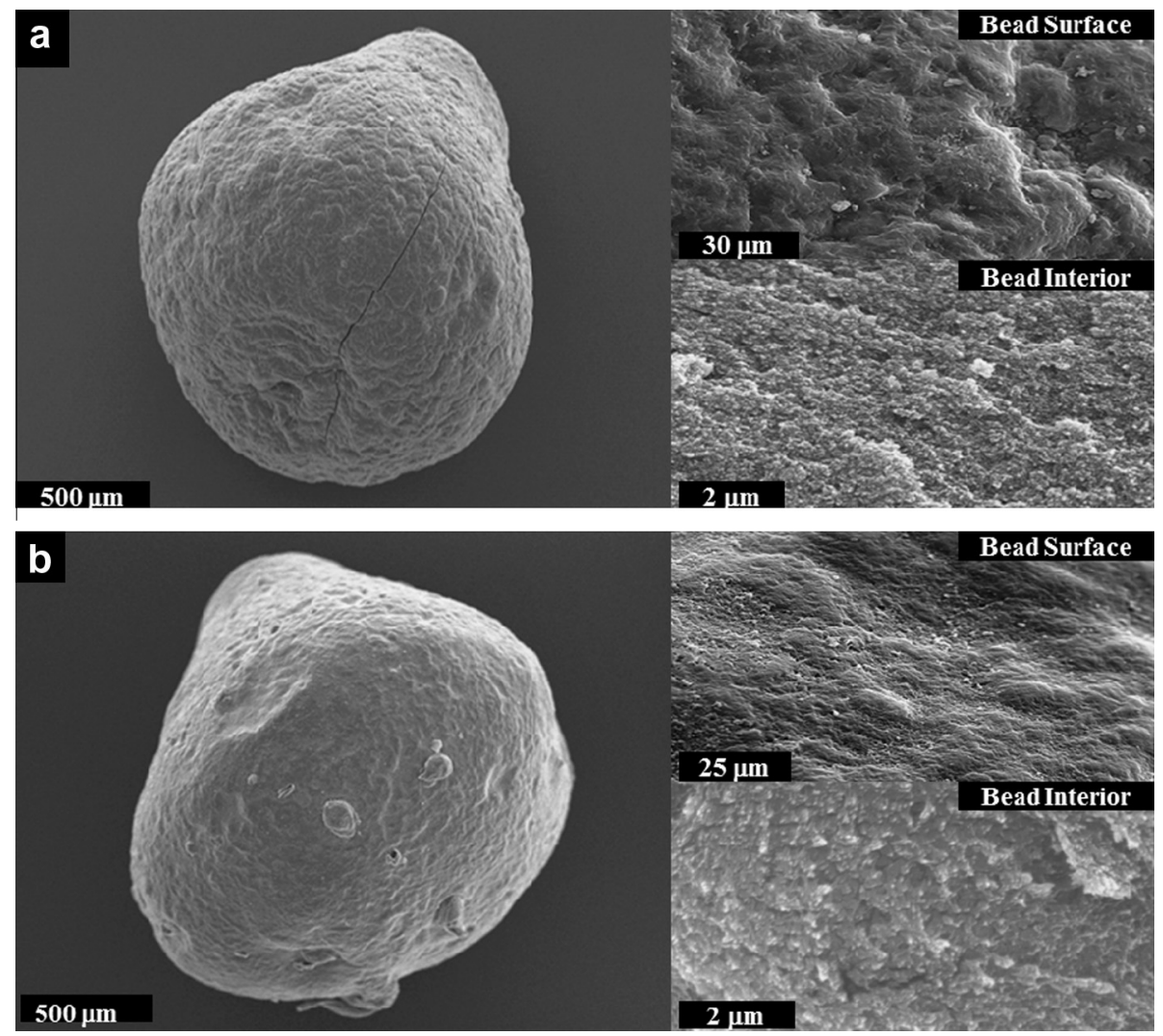

Fig. 1. SEM micrographs of (a) C beads (Chitosan) and (b) CG beads (Chitosan/Glyoxal).
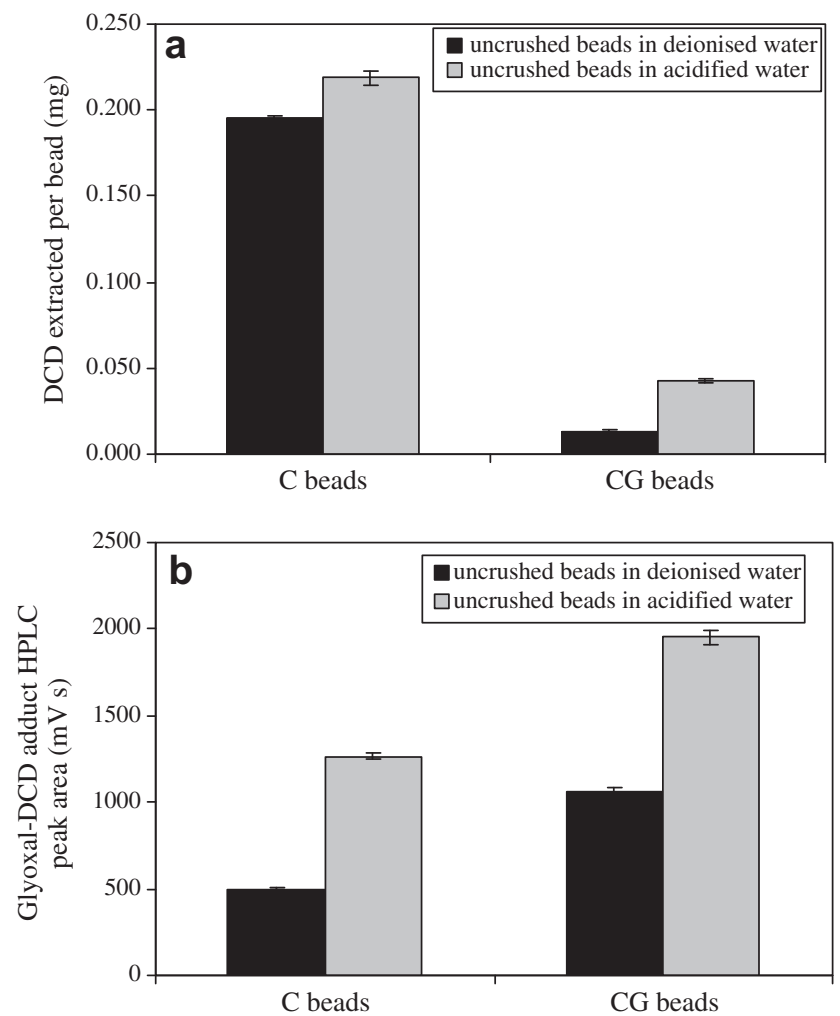

Fig. 2. Amount of (a) DCD (in $\mathrm{mg} \pm$ one deviation of the standard error of the mean (SE), $n=3$ ) and (b) glyoxal-DCD adduct (measured as a HPLC peak area in mV s) extracted from C (Chitosan) and CG (Chitosan/Glyoxal) beads following incubation in deionised or acidified water ( $\mathrm{pH}$ of 2.1). (All incubations carried out at $5{ }^{\circ} \mathrm{C}$ for $14 \mathrm{~d}$ with ten beads in $100 \mathrm{~mL}$.) consistent with the earlier observation that CG beads were $46 \%$ heavier than $C$ beads due to a higher glyoxal content. As it is only after acidification that the locked fraction of DCD was also released from the beads, it is likely that glyoxal derivatives (i.e. glyoxal-DCD adducts and glyoxal polymers/oligomers) trapped the locked DCD within the beads. Glyoxal is a very commonly used covalent crosslinker for chitosan CRFs, but its ability to undergo polymerisation and/or oligomerisation within chitosan beads has not been reported, nor the effect this may have on the release of the entrapped species within the polymeric matrix.

\subsubsection{DCD release into water}

The incubation of chitosan beads in deionised water resulted in a rapid release of DCD before it reached a plateau (Fig. 3a): C and CG beads lost 84 and 19\% of their total DCD content respectively after $9 \mathrm{~h}$. These values correspond to available DCD fractions held within the beads (Table SM-1). After $14 \mathrm{~d}$, the fractions of total DCD content released slightly increased to $87 \%$ and $23 \%$ for C and CG beads respectively, highlighting that glyoxal-crosslinked chitosan can retain a substantial proportion of its DCD content for long periods of time.

Conversely, the locked DCD fractions in C and CG beads represented 16 and $81 \%$ of the total DCD content respectively. Despite large differences in their total DCD content, this translated into $C$ and CG beads containing similar amounts of locked DCD (Table SM-1). It should be noted that a brief final washing of the beads with deionised water removed $32 \%$ and $17 \%$ of the total DCD content of $C$ and CG beads respectively, indicating that a substantial proportion of the available DCD fraction simply lies close to the outer surface of the beads.

At each incubation time, beads were removed from the deionised water, dried and weighed. A weight loss, determined by comparison with the initial (pre-incubation) dry weight, was 

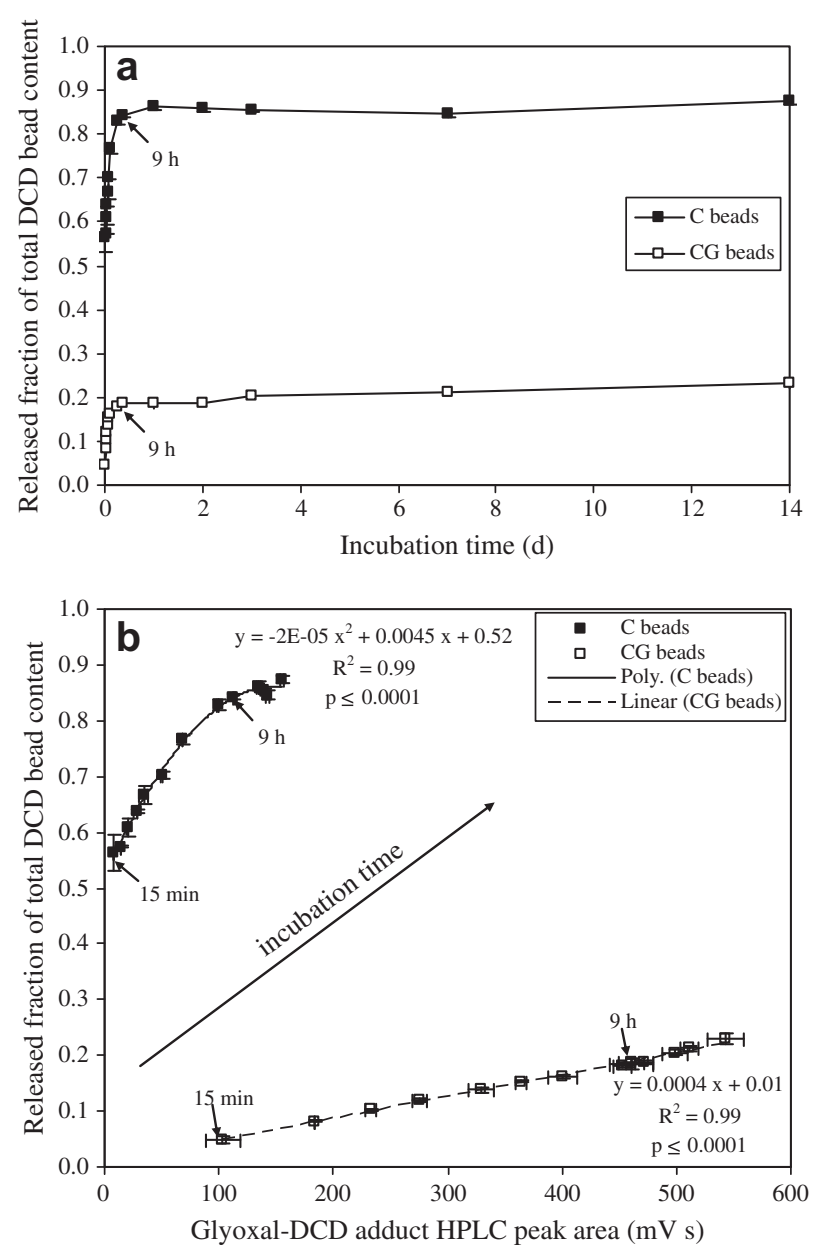

Fig. 3. Fraction of total $D C D$ bead content $( \pm S E, n=3)$ released from $C$ (Chitosan) and CG (Chitosan/Glyoxal) beads during incubation in deionised water, plotted against (a) incubation time (15 min up to $14 \mathrm{~d}$ ) and (b) glyoxal-DCD adduct HPLC peak area (in $\mathrm{mV} \mathrm{s} \pm \mathrm{SE}, n=3$ ) (All incubations carried out at $5{ }^{\circ} \mathrm{C}$ for $15 \mathrm{~min}$ up to $14 \mathrm{~d}$ with ten beads in $100 \mathrm{~mL}$ of deionised water; polynomial and linear regression lines fitted for C and CG beads respectively in (B)). observed. It was positively correlated with the HPLC peak area of the glyoxal-DCD adduct for C and CG beads $\left(R^{2}>0.9, p \leqslant 0.0001\right)$ (Fig. SM-1). The beads were therefore being eroded by releasing quantifiable amounts of a glyoxal-DCD adduct. Quite significantly, the release of the glyoxal-DCD adduct was positively correlated $\left(R^{2}=0.99, p \leqslant 0.0001\right)$ with that of DCD (Fig. 3b). It is not clear why both sets of beads behaved slightly differently (polynomial fitting for $C$ beads, linear fitting for CG beads).

\subsubsection{Swelling ratio}

The swelling ratio $\left(S_{w} \%\right)$ of CG and $\mathrm{C}$ beads was measured upon immersion in water as a function of time over a $14 \mathrm{~d}$ incubation. It was observed that the CG beads had a higher swelling ratio compared to the $C$ beads by $27 \%$ (Fig. SM-2). In both cases, most of the swelling took place over the first $9 \mathrm{~h}$ of immersion. This was unexpected because it had been anticipated that the increased amount of glyoxal polymers/oligomers in CG beads would block some pores and make it harder for water to diffuse in.

\subsection{5. $D C D$ release kinetics}

Kinetic analysis of DCD bead release was carried out during incubation in water over nine time points between $15 \mathrm{~min}$ and $9 \mathrm{~h}$ (Table SM-2). The Korsmeyer-Peppas model displayed the best fit among all four models tested for $C\left(R^{2}=0.97\right)$ and CG beads $\left(R^{2}=0.87\right)$. However, results should be read with caution as CG beads contained small amounts of DCD. The diffusional exponent $n$ values were lower than 0.5 suggesting that DCD was released by quasi-Fickian diffusion. Deviations from ideal Fickian diffusion could be explained by the existence of a bead erosion factor related to the release of glyoxal derivatives.

\section{2. $D C D$ release in soil}

The incubation of lightly washed $C$ beads $(0.148 \mathrm{mg}$ DCD per bead) in soil under simulated rainfall and soil moisture conditions resulted in the delayed release of DCD over $7 \mathrm{~d}$ (Fig. 4). DCD release in soil significantly increased with time $(p \leqslant 0.0001)$ : the amount of DCD recovered in soil was the lowest after $1 \mathrm{~d}$, followed by $4 \mathrm{~d}$ and then $7 \mathrm{~d}$ ( $p \leqslant 0.0001$ for all pairwise comparisons). Treatment had a significant effect on DCD soil release $(p \leqslant 0.0001)$ as it was

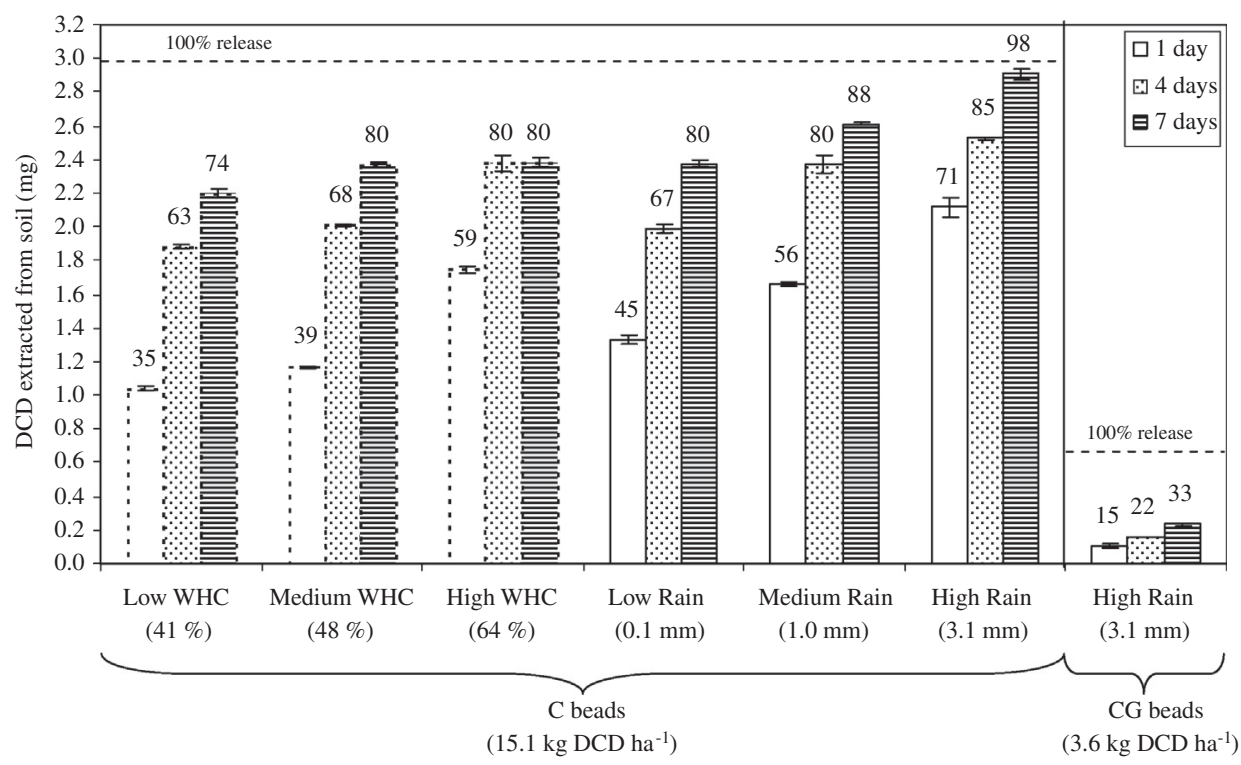

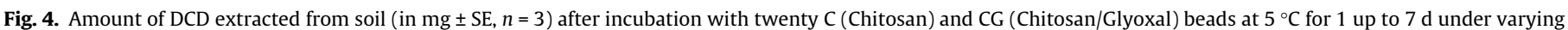

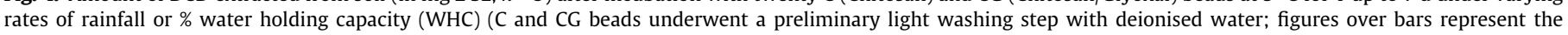
percentages of total DCD bead content released and recovered in soil). 
observed that rainfall caused more release than soil moisture (i.e. WHC treatment) alone. This most likely reflected the fact that the beads were wet more thoroughly and quickly when water was applied from above (rain) than when moisture had to diffuse up from the soil surface into the hydrogel. The treatment rate also showed a significant effect on DCD soil release $(p \leqslant 0.0001)$ : the amount of DCD in soil was greatest at the high levels of rainfall or WHC, followed by medium and then low levels $(p \leqslant 0.0005$ for all pairwise comparisons). The interaction between treatment rate and time was also significant ( $p=0.0187$ ).

The fractions of total DCD bead content released in soil by $C$ beads after $7 \mathrm{~d}$ were close to values of the available DCD fraction (i.e. 84\%), except in the high rain treatment where almost all the encapsulated DCD was released. This indicates that under low or moderate moisture, $C$ beads can quickly release a large portion if not all their available DCD fraction without depleting a significant proportion of the locked reservoir.

The behaviour of lightly washed CG beads ( $0.035 \mathrm{mg}$ DCD per bead) was different. The amount of DCD released in soil under the high rainfall treatment was much lower than with $C$ beads (Fig. 4). This was to be expected as CG beads contained about five times less DCD than $C$ beads. However, the fraction of total DCD bead content recovered in soil after $7 \mathrm{~d}$ was only $33 \%$ compared with $98 \%$ for the $C$ beads.

Consequently, nine times more DCD remained encapsulated in twenty CG beads ( $0.469 \mathrm{mg}$ DCD) than in twenty $\mathrm{C}$ beads $(0.051 \mathrm{mg}$ DCD) after $7 \mathrm{~d}$. This is in contrast to the release experiment in water, in which the actual amount of DCD remaining in both sets of beads was about the same after a $7 \mathrm{~d}$ incubation ( $0.033 \mathrm{mg}$ DCD per bead). These results suggest that CG beads could efficiently retain DCD after soil application under high rain conditions.

The fraction of total DCD bead content released by $\mathrm{C}$ and CG beads in soil after a $7 \mathrm{~d}$ incubation (98\% and 33\% respectively) was noticeably greater than during a $2 \mathrm{wk}$ incubation in deionised water (87\% and 23\% respectively in Section 3.1.3). This may be due to soil acidity ( $\mathrm{pH}$ of 5.5) that opens the chitosan mesh faster than under neutral $\mathrm{pH}$. This indicates that $\mathrm{C}$ beads could be less effective in retaining DCD than CG beads under some soil types.

\section{Conclusions}

It was found that $C$ beads (Chitosan) and CG beads (Chitosan/ Glyoxal) contained two fractions of DCD: one that was quickly released in water (available DCD) and one that was not (locked DCD). The content of the glyoxal held within the beads was key to control (i) the total amount of DCD encapsulated and (ii) the allotment between the available and the locked fraction. The higher the glyoxal content, the lower the total amount of DCD loaded in the beads, but the higher the proportion of locked DCD which led to a more delayed release in water or in soil. It is likely glyoxal derivatives (i.e. glyoxal-DCD adducts and glyoxal polymers/oligomers) trap the DCD within the beads. These results suggest that a combination of $C$ and CG beads could be used to fit two purposes: a quick release of DCD with $C$ beads necessary after fertiliser (urea or ammoniumbased) or slurry application, and a slower release of DCD with CG beads to sustain nitrification inhibition. Altering some parameters during the hydrogel synthesis process (e.g. concentration of the DCD solution, geometry of the hydrogel) could also modulate DCD soil release and limit the need for repeated and/or excessive usage of agrochemical like DCD. The $\mathrm{pH}$ of the surrounding media is one key factor in controlling the release profile of the locked fraction of DCD. Further studies should be carried out to investigate the effect of $\mathrm{pH}$ and of soil enzymes on the release of DCD from the beads.

\section{Acknowledgements}

The authors thank the Irish Department of Agriculture, Food and the Marine (DAFM) for funding this study (Research Stimulus Fund Programme - RSF 07545) and Dr. Jim Grant (Teagasc) for advice on statistics. The DAFM had no other role in this study.

\section{Appendix A. Supplementary material}

Supplementary data associated with this article can be found, in the online version, at http://dx.doi.org/10.1016/j.chemosphere. 2013.08.043.

\section{References}

Akelah, A., 1996. Novel utilisation of conventional agrochemicals by controlled release formulation. Mater. Sci. Eng. C - Mater. 4, 83-98.

Berger, J., Reist, M., Mayer, J.M., Felt, O., Peppas, N.A., Gurny, R., 2004. Structure and interactions in covalently and ionically crosslinked chitosan hydrogels for biomedical applications. Eur. J. Pharm. Biopharm. 57, 19-34.

Bishop, P.A., 2010. Polymer Coated Controlled Release Agrichemicals as Mitigation Tools in Pastoral Farming. PhD thesis, Massey University, Palmerston North, New Zealand.

Devi, N., Maji, T.K., 2009. Effect of crosslinking agent on neem (Azadirachta Indica A Juss.) seed oil (NSO) encapsulated microcapsules of $\kappa$-carrageenan and chitosan polyelectrolyte complex. J. Macromol. Sci. A 46, 1114-1121.

Di, H.J., Cameron, K.C., 2004. Treating grazed pasture soil with a nitrification inhibitor, eco- $\mathrm{n}^{\mathrm{TM}}$, to decrease nitrate leaching in a deep sandy soil under spray irrigation - a lysimeter study. New Zeal. J. Agric. Res. 47, 351-361.

Di, H.J., Cameron, K.C., Sherlock, R.R., 2007. Comparison of the effectiveness of a nitrification inhibitor, dicyandiamide, in reducing nitrous oxide emissions in four different soils under different climatic and management conditions. Soil Use Manage. 23, 1-9.

Estermaier, L.M., Sieber, A.H., Lottspeich, F., Matern, D.H.M., Hartmann, G.R., 1992 Biochemical degradation of cyanamide and dicyandiamide. Angew. Chem. Int Ed. 31, 620-622.

Fan, L., Jin, R., Le, X., Zhou, X., Chen, S., Liu, H., Xiong, Y., 2012. Chitosan microspheres for controlled delivery of auxins as agrochemicals. Microchim. Acta 176, $381-$ 387.

Fernández-Pérez, M., González-Pradas, E., Villafranca-Sánchez, M., Flores-Céspedes, F., 2001. Mobility of atrazine from alginate-bentonite controlled release formulations in layered soil. Chemosphere 43, 347-353.

Kelliher, F.M., Clough, T.J., Clark, H., Rys, G., Sedcole, J.R., 2008. The temperature dependence of dicyandiamide (DCD) degradation in soils: a data synthesis. Soil Biol. Biochem. 40, 1878-1882.

Loeffler, K.W., Koehler, C.A., Paul, N.M., De Haan, D.O., 2006. Oligomer formation in evaporating aqueous glyoxal and methyl glyoxal solutions. Environ. Sci. Technol. 40, 6318-6323.

Nakajima, K., Ohta, K., Mostefaoui, T.A., Chai, W., Utsukihara, T., Horiuchi, C.A. Murakami, M., 2007. Glyoxal sample preparation for high-performance liquid chromatographic detection of 2,4-dinitro-phenylhydrazone derivative: suppression of polymerization and mono-derivative formation by using methanol medium. J. Chromatogr. A 1161, 338-341.

Panicucci, R., McClelland, R.A., 1989. 4,5-Dihydro-4,5-dihydroxyimidazoles as products of the reduction of 2-nitroimidazoles. HPLC assay and demonstration of equilibrium transfer of glyoxal to guanine. Can. J. Chem. 67 2128-2134.

Quiñones, J.P., Coll García, Y., Curiel, H., Peniche Covas, C., 2010. Microspheres of chitosan for controlled delivery of brassinosteroids with biological activity as agrochemicals. Carbohyd. Polym. 80, 915-921.

Rinaudo, M., 2006. Chitin and chitosan: properties and applications. Prog. Polym. Sci. 31, 603-632.

Stark, C.H., Richards, K.G., 2008a. The continuing challenge of nitrogen loss to the environment in the context of global change and advancing research. Dyn. Soil Dyn. Plant 2, 1-12.

Stark, C.H., Richards, K.G., 2008b. The continuing challenge of nitrogen loss to the environment: environmental consequences and mitigation strategies. Dyn. Soil Dyn. Plant 2, 41-55.

Teixeira, M.A., Paterson, W.J., Dum, E.J., Li, Q., Hunter, B.K., Goosen, M.F.A., 1990 Assessment of chitosan gels for the controlled release of agrochemicals. Ind. Eng. Chem. Res. 29, 1205-1209.

Turowski, M., Deshmukh, B., 2004. Direct chromatographic method for determination of hydrogen cyanamide and dicyandiamide in aqueous solutions. Anal. Lett. 9, 1981-1989.

Watson, C.J., Laughlin, R.J., McGeough, K.L., 2009. Modification of nitrogen fertilisers using inhibitors: Opportunities and potentials for improving nitrogen use efficiency. In: Proceedings No. 658, International Fertiliser Society, York, UK, 39 pp

Yu, Q. Chen, Y, Ye, X Z Zhang Q Zhang, Z, Tian, P., 2007. Evaluation of nitrification inhibitor 3,4-dimethyl pyrazole phosphate on nitrogen leaching in undisturbed soil columns. Chemosphere 67, 872-878. 